\title{
ARBITRARILY LARGE CONTINUOUS ALGEBRAS ON ONE GENERATOR
}

BY

\author{
JIŘ́ ADÁMEK, VÁCLAV KOUBEK, EVELYN NELSON AND JAN REITERMAN
}

\begin{abstract}
Generation of order-continuous algebras is investigated for various concepts of continuity. For the continuity understood as the preservation of joins of countably-directed sets, arbitrarily large infinitary continuous algebras on one generator are constructed.
\end{abstract}

0. Introduction. Free continuous algebras have been explicitly described in [4] as algebras of trees for arbitrary (possibly infinitary) types of algebras and very general types of join-continuity, in a sense made precise in $\$ 1$ below. Usually, in dealing with mathematical objects which are set-based, if free objects exist, then their existence can be proved using the time-honoured construction of universal objects (see Bourbaki [8]) which depends on the formation of subobjects and products (which our algebras have), and on the property we call "bounded generation", that is, the cardinality of an object generated by a set $X$ is bounded by some cardinal depending only on the cardinality of $X$.

In this paper, we investigate the question of bounded generation for continuous algebras. We provide, in $\$ 2$, a large variety of cases in which these algebras do indeed have bounded generation. Nevertheless, there is, as proved in $\S 4$, a class of continuous algebras which does not have bounded generation, namely those algebras with one operation of countable rank, where continuity refers to the existence and preservation of joins of countably-directed sets. A strengthening of this result, presented in $\$ 5$, shows that the category of such algebras is not extremally co-wellpowered. To our knowledge, this is the only known example of an "algebraic" setting which does not have bounded generation but nevertheless has free objects.

These results depend on set-theoretical assumptions which are discussed in $\S 3$. The construction, in $\S 4$, of a continuous algebra of power $\beta$, on one generator, depends on the existence of a binary tree of $\operatorname{depth} \beta$ with leaves at all infinite levels. In $\S 3$, we give a characterization of those cardinals $\beta$ for which such a tree exists; they include all cardinals which are below the first strongly inaccessible. In particular, if there are no strongly inaccessible cardinals, then we obtain unbounded generation. We do not know whether unbounded generation can be proved for these,

Received by the editors March 14, 1984 and, in revised form, January 16, 1985. 1980 Mathematics Subject Classification. Primary 08C05, 06F99.

Key words and phrases. Continuous algebras, unbounded generation, subtle cardinals.

(1985 American Mathematical Society $0002-9947 / 85 \$ 1.00+\$ .25$ per page 
or other, continuous algebras, with no set-theoretical assumptions, or whether the presence of a proper class of measurable cardinals implies bounded generation.

The authors express their thanks to Donald Monk, Donald Pelletier and James Schmerl for providing helpful information about subtle cardinals. Also, financial support of the National Science and Engineering Research Council of Canada and of the Science and Engineering Research Board of McMaster University is gratefully acknowledged.

\section{Preliminaries.}

1.1. Z-completeness and Z-continuity. We use the concept of a subset system $Z$ introduced by ADJ [5]: $Z$ is a map assigning to each poset $P$ a collection $Z(P)$ of subsets of $P$ in such a way that for any order-preserving map $f: P \rightarrow Q$, if $X \in Z(P)$ then $f(X) \in Z(Q)$. For example, each ordinal $\alpha$ defines a subset system $\alpha$ such that $\alpha(P)$ is the collection of all $\alpha$-chains in $P($ i.e., all images of order-preserving maps $\alpha \rightarrow P$ ).

A poset $P$ is called $Z$-complete if each set $X \in Z(P)$ has a join $\vee X$ in $P$. An order-preserving map $f: P \rightarrow Q$ is called $Z$-continuous if it preserves the join of any $X \in Z(P)$, i.e., $f(\vee X)=\bigvee f(X)$.

Let $\Sigma$ be a type of algebra, i.e., a set together with a map assigning to each $\sigma \in \Sigma$ a cardinal number ar $\sigma$ (the arity of $\sigma$ ). An algebra of type $\Sigma$ consists of a set $A$ and a collection of operations $\sigma: A^{n} \rightarrow A$ for all $\sigma \in \Sigma$, ar $\sigma=n$.

A $Z$-continuous algebra is an algebra $A$ endowed with a partial order such that $A$ is a $Z$-complete poset and each operation $\sigma: A^{n} \rightarrow A(\sigma \in \Sigma)$ is order-preserving and preserves joins of all nonempty sets in $Z\left(A^{n}\right)$ (where $A^{n}$ is ordered componentwise).

EXAMPLES. (i) $\Delta$ : for each poset $P, \Delta(P)$ is the set of all (upwards) directed subsets of $P$. A $\Delta$-continuous algebra is a poset $A$ which is $\Delta$-complete (i.e., has joins of directed subsets, including the least element $\bigvee_{\phi}$ ) together with operations $\sigma: A^{n} \rightarrow A$ preserving nonempty directed joins.

(ii) $\Delta_{\omega}$ : for each poset $P, \Delta_{\omega}(P)$ is the set of all countably-directed subsets, i.e., subsets $M \subseteq P$ such that any countable set $M_{0} \subseteq M$ has an upper bound in $M$. A $\Delta_{\omega}$-continuous algebra is a $\Delta_{\omega}$-complete poset $A$ together with operations $\sigma: A^{n} \rightarrow A$ preserving nonempty $\omega$-directed joins.

REMARK. The requirement that the operations preserve just the nonempty Z-joins stems from computer science (see [4]). We can also study algebras in which operations are $Z$-continuous. If $\phi \subseteq Z$, i.e., if $\phi \in Z(P)$ for each poset $P$, this means that the least element $\perp$ (which exists in each $Z$-complete poset) is an idempotent element of all operations. No result of the present paper depends on this detail: we work with continuous algebras as defined above, but when constructing a continuous algebra, $\perp$ will always be an idempotent.

If $\phi \nsubseteq Z$, then the operations of a $Z$-continuous algebra are $Z$-continous maps, of course.

1.2. Bounded generation. A subalgebra $B$ of a $Z$-continuous algebra $A$ is a subset $B \subseteq A$ closed under the operations (i.e., $\sigma\left(B^{n}\right) \subseteq B$ for each $\sigma \in \Sigma$ of arity $n$ ) and $Z$-joins (i.e., $\mathrm{V} X \in B$ for each $X \in Z(A), X \subseteq B$ ). A set $X \subseteq A$ is said to generate $A$ if for each subalgebra $B$ with $X \subseteq B$ we have $A=B$. 
We also use the concept of a weak subalgebra of a $Z$-continuous algebra $A$ : this is a $Z$-continuous algebra $B$ with $B \subseteq A$ such that the inclusion map $B \rightarrow A$ is a $Z$-continuous homomorphism. This means that $B$ is closed under the operations (and the operations of $B$ are restrictions of those of $A$ ) and that the order of $B$ is weaker than that of $A$ but such that the joins $\bigvee X$ in $A$ and $B$ coincide for each $X \in Z(B)$.

Definition. Let $Z$ be a subset system. We say that $Z$-continuous algebras of a given type have bounded generation if for each cardinal $n$ there exists a cardinal $k$ such that any $Z$-continuous algebra generated by an $n$-element set has cardinality at most $k$.

REMARK. A set $X$ generates a $Z$-continuous algebra $A$ if $A=\bar{X}_{i}$ for some ordinal $i$, where we define subposets $X_{i} \subseteq \bar{X}_{i} \subseteq A$ by the following induction.

(i) $X_{0}=X$ and

$$
\bar{X}_{0}= \begin{cases}X_{0} & \text { if } \phi \nsubseteq Z, \\ X_{0} \cup\{\perp\} & \text { if } \phi \subseteq Z ;\end{cases}
$$

(ii) For $i \geqslant 1, \bar{X}_{i}$ is the $Z$-closure of $X_{i}$, i.e., the least subset of $A$ which contains $X_{i}$ and fulfills $\vee Y \in \bar{X}_{i}$ for each $Y \in Z(A)$ with $Y \subseteq \bar{X}_{i}$; $\left.\bar{X}_{i}\right)$;

(iii) $X_{i+1}$ is the operational hull of $\bar{X}_{i}$ (i.e., the nonordered subalgebra generated by

(iv) $X_{i}=\cup_{j<i} X_{j}$ for each limit ordinal $i$.

We say that $Z$-continuous algebras have $k$-generation for an ordinal $k$ provided that $A=\bar{X}_{k}$ for each set $X$ of generators of a $Z$-continuous algebra $A$. If this is the case, generation is obviously bounded: for each $i$ we have

(1) card $\bar{X}_{i} \leqslant 2^{\text {card } X_{i}}$ because there are at most card $2^{X_{i}}$ subsets of $X_{i}$ and

(2) card $X_{i+1} \leqslant$ card $\bar{X}_{i}^{q}$, where $q$ is any infinite cardinal larger than the sum of all arities.

Generation is bounded for an impressive collection of types and subset systems. For example, generation of finitary algebras is always bounded (see [11]). We discuss this in the next part of the present paper. Nevertheless, generation of infinitary $\Delta_{\omega}$-continuous algebras is not bounded. This will be proved in $\S 4$, under set-theoretical restrictions discussed in $\S 3$.

1.3. Bounded strong generation. We also investigate a stronger concept of generation:

Definition. A $Z$-continuous algebra $A$ is said to be strongly generated by a subposet $X \subseteq A$ if we have $B=A$ for each weak subalgebra $B$ containing $X$ as a subposet. We say that $A$ has $n$ strong generators for $n=$ card $X$.

REMARKs. (i) The intuitive meaning of strong generation is that not only all points of $A$ are obtained by a successive application of operations and $Z$-joins (to all previously obtained points), but also the ordering of $A$ is determined by the ordering of $X$. The ordering of $X$ "forces" an ordering of the results of operations (since $\sigma\left(a_{i}\right) \leqslant \sigma\left(b_{i}\right)$ whenever $a_{i} \leqslant b_{i}$ for each $\left.i\right)$ and on the results of $\Delta_{\omega}$-joins (since $\mathrm{V} a_{i} \leqslant \mathrm{~V} b_{i}$ whenever $a_{i} \leqslant b_{i}$ for each $i$ ) and using this successively, we "force" all the order of $A$. 
(ii) We say that Z-continuous algebras have bounded strong generation if for each cardinal $n$ there exists a cardinal $k$ such that any $Z$-continuous algebra on $n$ strong generators has power $\leqslant k$.

1.4. Categorical remarks. Although no category theory is used in our paper, we want to make some comments on the categorical background of the results below. Let $Z$-Alg denote the category of $Z$-continuous algebras (of a fixed type) and $Z$-continuous homomorphisms. This is a complete, well-powered category and its limits are preserved by the forgetful functor $U: Z$-Alg $\rightarrow$ Set. All of this is easy to prove.

(a) Free algebras. The forgetful functor $U$ is an adjoint because any set generates a free $Z$-continuous algebra. This has been proved in [4] for all $Z$, and earlier, for special cases, in $[\mathbf{2}, \mathbf{5}, \mathbf{1 1}]$, by giving an explicit construction of the free algebras. The proofs were rather technical.

In case generation is bounded, the existence of free algebras follows immediately from the Adjoint Functor Theorem [9] because all algebras generated by a set $X$ form an (essentially small) solution set. If generation is not bounded, for example in case $Z=\Delta_{\omega}$, there seems to be no "categorical reason" for the existence of free algebras.

Let us remark that free infinitary algebras fail to exist if instead of $Z$-continuity of operations we require separate $Z$-continuity (see [3]). An operation $\sigma: A^{n} \rightarrow A$ is separately $Z$-continuous if for each $\left(a_{i}\right)_{i<n} \in A^{n}$ we have

$$
\bigvee_{x \in X} \sigma\left(a_{0}, a_{1}, \ldots, a_{i-1}, x, a_{i+1}, \ldots\right)=\sigma\left(a_{0}, a_{1}, \ldots, a_{i-1}, a_{i}, a_{i+1}, \ldots\right)
$$

provided that $X \in Z(A)$ has join $a_{i}$.

(b) Co-well-poweredness. Recall that an extremal epi is an epi $f: A \rightarrow A^{\prime}$ such that if $f=m \cdot e$ for a mono $m: B \rightarrow A^{\prime}$, then $m$ is an isomorphism.

The category Z-Alg is extremally co-well-powered iff it has bounded strong generation. In fact,

(i) if Z-Alg is extremally co-well-powered, we use the fact that if $X$ is a set of strong generators of $A$, then the canonical morphism from the free algebra $F(X)$ into $A$ is an extremal epi;

(ii) if $Z$-Alg has bounded strong generation, then for each extremal epi $f: A \rightarrow A^{\prime}$ we use the fact that $f(A)$ strongly generates $A^{\prime}$ : if a weak subalgebra $B$ of $A^{\prime}$ contains $f(A)$, we can restrict $f$ to a $Z$-continuous homomorphism $e: A \rightarrow B$, and the inclusion map $m: B \rightarrow A^{\prime}$ is a mono with $f=m \cdot e$.

Consequently, the category $\Delta_{\omega}$-Alg is not co-well-powered, in fact, not even extremally co-well-powered.

It is easy to prove that if Z-Alg is co-well-powered, then it has bounded generation.

A. Pasztor [12] studies categories of ordered algebras which are not necessarily $Z$-complete, and $Z$-continuous homomorphisms, and proves that these categories are also not co-well-powered in general. For example, consider $Z=\Delta$ and the type with one unary operation $\sigma$. Then for each limit ordinal $\alpha$ the inclusion $\omega \rightarrow \alpha$, where $\alpha$ has the usual order and $\sigma(i)=i+1$, is an epimorphism. 
(c) Colimits. If strong generation is bounded, then Z-Alg is co-complete. This follows from the form of the Adjoint Functor Theorem presented in [9, Theorem 28.10].

1.5. Open problems. (i) Is the category $\Delta_{\omega}$-Alg co-complete for any infinitary type of algebras?

(ii) Is generation of $\Delta_{\omega}$-continuous algebras unbounded without any additional assumptions on set theory?

(iii) Is there a subset system $Z$ such that $Z$-continuous algebras have bounded strong generation but unbounded generation?, or they have bounded generation but Z-Alg is not co-well-powered?

REMARK. If the answer to (i) is negative, an important example of a non-cocomplete category of monad algebras (over the category of $\Delta_{\omega}$-complete posets and $\Delta_{\omega}$-continuous maps) is obtained. In the first example of this sort the monad was artificial (see [1]), and here we have a "natural" one.

2. Criteria ensuring bounded generation. In the present section we exhibit several criteria for $Z$-continuous algebras to have bounded generation. Recall that $\alpha$ is the subset system of all $\alpha$-chains. We write $\alpha \subseteq Z$ if each $\alpha$-chain in each $Z$-complete poset has a join, and $Z$-continuous maps preserve $\alpha$-joins.

A subset system $Z$ with $\phi \nsubseteq Z$ has 1 -generation (see 1.2) iff for each $Z$-continuous algebra $A$ and each subset $B$ closed under the operations, the $Z$-closure $\bar{B}$ of $B$ is closed under the operations. (Put $X_{1}=B$.) If $\phi \subseteq Z$, then $Z$ has 1-generation iff, for each subset $B \subseteq A$ with $\perp \in B$ (where $\perp=\bigvee_{\phi}$ ) which is closed under the operations, $\bar{B}$ is also closed under the operations.

Proposition 1. Z-continuous algebras have 1-generation for each subset system $Z$ such that every chain of cofinality $n<$ ar $\sigma$ is a Z-set for each operation $\sigma$.

Proof. Let $A$ be a $Z$-continuous algebra, and let $B \subseteq A$ be closed under the operations. Assuming that $\phi \nsubseteq Z$ or $\perp \in B$, we prove that $\bar{B}$ is also closed under the operations. For each $\sigma \in \Sigma$ of arity $k$ and arbitrary $\left(x_{i}\right)_{i<k} \in \bar{B}^{k}$ we prove that $\sigma\left(x_{i}\right)_{i<k} \in \bar{B}$. We prove by induction on $n \leqslant k$ that for arbitrary $\left(y_{i}\right)_{i<k} \in B^{k}$ we have

$$
\sigma\left(x_{0}, x_{1}, \ldots, x_{i}, \ldots, y_{n}, y_{n+1}, \ldots, y_{j}, \ldots\right) \in \bar{B} .
$$

For $n=k$ this concludes the proof.

(a) $n=0$ : since $B$ is closed under $\sigma$, we have $\sigma\left(y_{0}, y_{1}, \ldots,\right) \in B$.

(b) $n+1:$ define a map $f: A \rightarrow A$ by

$$
f(z)=\sigma\left(x_{0}, x_{1}, \ldots, x_{i}, \ldots, z, y_{n+1}, y_{n+2}, \ldots, y_{j}, \ldots\right) .
$$

Assuming that the statement holds for $n$, i.e., that $f(B) \subseteq \bar{B}$, we shall prove that $\bar{B} \subseteq f^{-1}(\bar{B})$, in particular, $f\left(x_{n}\right) \in \bar{B}$. It is sufficient to verify that the set $f^{-1}(\bar{B})$ has the following property:

$$
\bigvee Y \in f^{-1}(\bar{B}) \text { for each } Y \in Z(A) \text { with } Y \subseteq f^{-1}(\bar{B})
$$


Since $\bar{B}$ is the least super-set of $B$ with this property, we conclude then that $\bar{B} \subseteq f^{-1}(\bar{B})$. If $Y=\phi$, then $\phi \subseteq Z$ and hence, $\vee Y=\perp \in B$ by assumption-it follows that $\vee Y \in f^{-1}(\bar{B})$ by the induction hypothesis. If $Y \neq \phi$, then $f$ clearly preserves the join of $Y$ (since $\sigma$ preserves nonempty $Z$-joins) and thus $f(\vee Y)=$ $\vee f(Y) \in \bar{B}$.

(c) $n$ a limit ordinal: let $\sigma\left(x_{0}, \ldots, x_{i}, \ldots, y_{m}, \ldots, y_{j}, \ldots\right)$ be in $\bar{B}$ for each $m<n$. For each $i<n$ choose $z_{i} \in B$ with $z_{i} \leqslant x_{i}$ (such $z_{i}$ clearly exists, since $x_{i} \in \bar{B}$ ) and put for each $m<n$,

$$
a_{m}=\sigma\left(x_{0}, x_{1}, \ldots, x_{i}, \ldots, z_{m}, z_{m+1}, \ldots, z_{u}, \ldots, y_{n}, y_{n+1}, \ldots, y_{j}, \ldots\right) .
$$

Since $\sigma$ is order-preserving, $m \leqslant m^{\prime}$ implies $a_{m} \leqslant a_{m^{\prime}}$. We have

$$
\bigvee_{m<n} a_{m}=\sigma\left(x_{0}, \ldots, x_{i}, \ldots, y_{n}, \ldots, y_{j}, \ldots\right)
$$

and this is a join of an $n$-chain in $A$ with $a_{m} \in \bar{B}, m<n$. Since $n \leqslant k=\operatorname{ar} \sigma$, by hypothesis $\mathbf{n} \subseteq Z$ and hence, $\vee_{m<n} a_{m} \in \bar{B}$. This concludes the proof.

COROLlaRY. Z-continuous algebras have 1-generation if

(i) all arities are finite, or

(ii) all arities are countable and $\omega \subseteq Z$, or

(iii) $\mathbf{n} \subseteq Z$ for each ordinal $n$.

PROPOSITION 2. If cardinalities of all Z-sets are bounded, then Z-continuous algebras of any type have bounded generation.

Proof. Let $n$ be a cardinal with card $X<n$ for any poset $P$ and any $X \in Z(P)$, and let $m$ be the least infinite regular cardinal larger than all arities and with $n \leqslant m$.

Given a $Z$-continuous algebra $A$ and a generating set $X \subseteq A$, we define subposets $X_{i} \subseteq \bar{X}_{i} \subseteq A$ for all ordinals $i$ as in the Remark in 1.2.

It is sufficient to prove that $A=X_{m}$. Evidently, $X_{m}=\bigcup_{j<m} X_{j}$ is closed under the operations in $A$. It is also closed under $Z$-joins: for each $M \in Z(A)$ with $M \subseteq X_{m}$ there exists $j<m$ with $M \subseteq X_{j}$ (because card $M<n \leqslant m$ ) and hence, $\vee M \in \bar{X}_{j} \subseteq$ $X_{j+1} \subseteq X_{m}$. Thus, $X_{m}$ is a subalgebra of $A$ containing $X$ and hence, $X_{m}=A$.

Definition. A subset system $Z$ is simple if it is

(i) productive: If $X_{i} \in Z\left(P_{i}\right)$ for $i \in I$, then $\prod_{i \in I} X_{i} \in Z\left(\prod_{i \in I} P_{i}\right)$, where $\Pi P_{i}$ is ordered component-wise;

(ii) associative: Given a $Z$-complete poset $P$ and $X \subseteq P$, the set $\tilde{X}=\{\bigvee Y \mid Y \in$ $Z(P)$ and $Y \subseteq X\}$ fulfills $\vee Y \in \tilde{X}$ for any $Y \in Z(P)$ with $Y \subseteq \tilde{X}$.

PROPOSITION 3. Z-continuous algebras of any type have 1-generation for each simple subset system $Z$ with $\phi \nsubseteq Z$.

Proof. Let $B \subseteq A$ as in Proposition 1. For each $\sigma \in \Sigma$ and $x_{i} \in \bar{B}(i<k=\operatorname{ar} \sigma)$ we are to show that $\sigma\left(x_{i}\right)_{i<k} \in \bar{B}$. Since $Z$ is simple, for each $i$ we have $X_{i} \in Z(A)$ with $X_{i} \subseteq B$ and $x_{i}=\vee X_{i}$. The set $\prod_{i<k} X_{i} \subseteq A^{k}$ is nonempty (because $\phi \nsubseteq Z$ ), and it is a $Z\left(A^{k}\right)$-set (because $Z$ is simple). Thus,

$$
\sigma\left(x_{i}\right)_{i<k}=\sigma\left(\bigvee \prod_{i<k} X_{i}\right)=\bigvee \sigma\left(\prod_{i<k} X_{i}\right)
$$


and the last join is in $\bar{B}$ because we have $\sigma\left(y_{i}\right)_{i<k} \in B \subseteq \bar{B}$ for any $y_{i} \in X_{i} \subseteq B$ $(i<k)$.

Example. Let $\mathbf{P}$ be the subset system of complete join semilattices: $\mathbf{P}(P)=\exp P$. Generation of $\mathbf{P}$-continuous algebras is bounded. This follows from the fact that $Z=\mathbf{P}-\boldsymbol{\phi}$ is simple.

Remark. Productivity itself is not sufficient: the subset system $\Delta_{\omega}$ is productive and yet $\Delta_{\omega}$-continuous algebras do not have bounded generation in general, as we prove in $\S 4$.

3. Leaves on trees. The construction of continuous algebras presented in the subsequent section is based on binary trees with leaves of all infinite depths. In the present section, we discuss the existence of such trees: If there do not exist too many strongly inaccessible cardinals, we present arbitrarily large such trees; on the other hand, if $\alpha$ is a measurable cardinal, then no such tree has depth $\alpha$.

Recall that a tree is a poset $T$ such that

(a) $T$ has a least element, called the root;

(b) for each $t \in T$, the subposet $\{s \in T \mid s<t\}$ is well ordered; its ordinal type is called the depth of $t$;

(c) $T$ is conditionally complete, i.e., each subset with an upper bound has a join.

We denote by $T(i)$ the set of all elements of depth $i$. Elements which have no successors are called leaves. The depth of $T$ is $\bigvee\{i \mid T(i) \neq \phi\}$.

A tree $T$ is binary if each element is either a leaf, or it has exactly 2 immediate successors ( $n$-ary trees are defined analogously). An important example is the uniform binary tree of depth $\beta$, characterized by the property that no element of depth $<\beta$ is a leaf. We can describe it as $U=\bigcup_{n \leqslant \beta} 2^{n}$, where $2^{n}$ is the set of all functions from $n$ to $2=\{0,1\}$, and the ordering of $U$ is set inclusion: Given $f \in 2^{n}$ and $g \in 2^{m}$,

$$
f \leqslant g \quad \text { iff } \quad n \leqslant m \text { and } f(i)=g(i) \text { for each } i \in n .
$$

The unique element of $2^{0}$ is the root of $U$. Each $f \in 2^{\beta}$ is a leaf, and each $f \in 2^{n}$ $(n<\beta)$ has two immediate successors: $f_{0}$ and $f_{1} \in 2^{n+1}$ extending $f$ by $f_{i}(n)=i$.

Clearly, each binary tree of depth $\leqslant \beta$ is isomorphic to a subtree of $U$. Analogously, each $m$-ary tree of depth $\leqslant \beta$ is isomorphic to a subtree of the uniform $m$-ary tree $\bigcup_{n \leqslant \beta} m^{n}$. Before turning to the existence of many-leaved trees, we prove that the amount of leaves of a given depth is not important:

Proposition 4. For each binary tree $S$ there exists a binary tree $\bar{S}$ with card $2^{n}$ leaves of depth $n+k$ for each limit ordinal $n$ such that $S(n)$ contains a leaf, and each finite $k$.

Proof. (I) We can disregard the $k$ 's. More precisely, it is sufficient to present a binary tree $\tilde{S}$ with card $2^{n}$ leaves in $\tilde{S}(n)$ for each $n \in M$, where

$$
M=\{n \mid n \text { a limit ordinal with a leaf in } S(n)\} .
$$

Then $\bar{S}$ is obtained by the following extension of $\tilde{S}$ : for each $n \in M$ we choose a disjoint decomposition $L_{n, k}(k<\omega)$ of the set of all leaves in $\tilde{S}(n)$ with card $L_{n, k}=$ card $2^{n}$, and we extend each leaf in $L_{n, k}$ by an arbitrary binary tree of depth $k$. 
(II) Denote by $\beta$ the depth of $S$, and let $U$ be the uniform binary tree of depth $\beta$. The set

$$
T=\bigcup_{n \leqslant \beta} S(n) \times U(n)
$$

ordered component-wise is clearly a quaternary tree such that $(s, u) \in T$ is a leaf iff $s$ is a leaf in $S$. The subset $\tilde{U}=\left\{f \in U \mid f \in 2^{n+2 k}\right.$ for some limit ordinal $n$ or $n=0$, and $k<\omega\}$ is a uniform quaternary tree: each $f \in 2^{n+2 k}$ has exactly four immediate successors in $2^{n+2 k+2}$. Thus, we can assume that $T$ is a subposet of $\tilde{U}$. Put

$$
\tilde{S}=\{s \in U \mid s \subseteq t \text { for some } t \in T\} .
$$

This is a subtree of the uniform tree $U$. For each $n \in M$ we have a leaf $s \in S(n)$ and for each $u \in U(n),(s, u) \in T$ is a leaf of $\tilde{S}$. The number of these leaves is $\operatorname{card} U(n)=\operatorname{card} 2^{n}$.

COROLlaRY. If there is a binary tree with leaves of all depths $n$ with $\alpha \leqslant n \leqslant \beta$ for a limit ordinal $\alpha$, then there is a binary tree with $2^{n}$ leaves of depth $n$ for all these $n$.

Next, we present a necessary and sufficient condition on a cardinal $\beta$ for the existence of a binary tree with leaves of all infinite depths $\leqslant \beta$. It turns out that the binarity is no important restriction: $\omega$-nary trees would be just as general, or $n$-ary trees for any cardinal $n$ such that no cardinal $\leqslant n$ is strongly inaccessible. Recall that an uncountable cardinal $\alpha$ is strongly inaccessible if it is uncountable, regular (i.e., not a sum of fewer than $\alpha$ cardinals which are all smaller than $\alpha$ ), and if $\beta<\alpha$ implies $2^{\beta}<\alpha$. It is consistent with set theory to assume that no cardinal is strongly inaccessible; for our trees we need a much weaker set-theoretical assumption. We call a tree $S$ bounded if the power of each $S(i)$ is smaller than any strongly inaccessible cardinal $\gamma>i$. Then $n$-ary trees mentioned above are bounded: if $\gamma>i$ is strongly inaccessible, then $\gamma>n$ and hence, $\gamma>n^{i} \geqslant \operatorname{card} S(i)$.

DeFinition [7]. A cardinal $\beta$ is subtle if

(*) for each closed cofinal set $M \subseteq \beta$ and each map $f$ assigning to every $\gamma \in M$ a set $f(\gamma) \subseteq \gamma$, there exist $\gamma<\gamma^{\prime}$ in $M$ with $f(\gamma)=f\left(\gamma^{\prime}\right) \cap \gamma$.

REMARKs. (1) Subtle cardinals are pretty large: each subtle cardinal is strongly inaccessible and the join of the strongly inaccessibles below it (see Baumgartner [7]). Consequently, if there are not "too many" strongly inaccessibles, then no cardinal is subtle. On the other hand, each measurable cardinal is subtle (see, for example, Kanamori and Magidor [10]).

(2) Each limit ordinal $\beta$ satisfying $(*)$ is a regular cardinal, and hence subtle: if $\beta$ is not a regular cardinal, then we have $\delta=\operatorname{cof} \beta<\beta$. There is a $\delta$-chain of ordinals $\alpha_{i}(i<\delta)$ such that $\beta=\bigvee_{i<\delta} \alpha_{i}$ and $\delta<\alpha_{i}<\beta$, and for each limit ordinal $j<\delta$ we have $\alpha_{j}=\mathrm{V}_{i<j} \alpha_{i}$. Then the set $M=\left\{\alpha_{i} \mid i<\delta\right\}$ is closed and cofinal, and the function $f$ defined by $f\left(\alpha_{i}\right)=\{i\}$ shows that $(*)$ does not hold.

(3) The proof of (ii) $\rightarrow$ (iii) in the following theorem is essentially due to D. Monk and J. Schmerl. 
THEOREM 1. For each cardinal $\beta$, the following are equivalent:

(i) There is a binary tree with leaves of all infinite depths $\leqslant \beta$.

(ii) There is a bounded tree with leaves of all infinite depths $\leqslant \beta$.

(iii) No cardinal $\leqslant \beta$ is subtle.

Proof. (i) $\rightarrow$ (ii) is clear.

(ii) $\rightarrow$ (iii) It is sufficient to prove that if a bounded tree $S$ has a leaf $s(\gamma) \in S(\gamma)$ for each $\gamma$ with $\omega \leqslant \gamma \leqslant \beta$, then $\beta$ is not subtle. By remark (ii), we can assume that $\beta$ is a join of strongly inaccessibles-else, $\beta$ cannot be subtle. The set $M$ of all cardinals $<\beta$ which are joins of strongly inaccessibles is closed and cofinal. We present a map $f$ with $f(\gamma) \subseteq \gamma$ for each $\gamma \in M$ and such that $f(\gamma) \neq f\left(\gamma^{\prime}\right) \cap \gamma$ for all $\gamma<\gamma^{\prime}$ in $M$.

There clearly exists a well-order $\sqsubseteq$ on $S$ such that $s \sqsubseteq s^{\prime}$ whenever $s$ has smaller depth than $s^{\prime}$, and $s(\gamma)$ is the first element of $S(\gamma)$ for each $\gamma$ with $\omega \leqslant \gamma \leqslant \beta$; denote by $s_{i}$ the $i$ th element in the order $\sqsubseteq$. Then $s(\gamma)=s_{\gamma}$ for each strongly inaccessible $\gamma<\beta$. In fact, let $s(\gamma)=s_{i}$, then $i \geqslant \gamma$ because $s(\gamma)$ has depth $\gamma$. Assuming $i>\gamma$, we get a contradiction: the node $s_{\gamma}$ has depth $\delta_{0}<\gamma$, but since $S$ is bounded, we have card $\bigcup_{\delta \leqslant \delta_{0}} S(\delta)<\gamma$.

The desired function $f$ is defined as follows:

$$
f(\gamma)=\left\{i \mid s_{i}<s(\gamma)\right\} \quad \text { for } \gamma \in M .
$$

We have $f(\gamma) \subseteq \gamma$ : let $\gamma=\bigvee \gamma_{k}$ with each $\gamma_{k}$ strongly inaccessible. Then $s_{i}<s(\gamma)$ implies $s_{i} \in S(\delta)$ for some $\delta<\gamma$, and then $\delta<\gamma_{k}$ for some $k$. We conclude that $s_{i} \sqsubseteq s\left(\gamma_{k}\right)=s_{\gamma_{k}}$ and hence, $i<\gamma_{k} \leqslant \gamma$. Further, $f(\gamma)=f\left(\gamma^{\prime}\right) \cap \gamma$ implies $\gamma=\gamma^{\prime}$ because

$$
s(\gamma)=\bigvee_{s<s(\gamma)} s=\bigvee_{i \in f(\gamma)} s_{i} \leqslant \bigvee_{i \in f\left(\gamma^{\prime}\right)} s_{i}=s\left(\gamma^{\prime}\right)
$$

and hence, $s(\gamma)=s\left(\gamma^{\prime}\right)$ since $s(\gamma)$ is a leaf.

(iii) $\Rightarrow$ (i) We prove this result by induction for all ordinals $\beta \geqslant \omega$. The case $\beta=\omega$ is clear, and from $\beta$ to $\beta+1$ it suffices to use Proposition 4 .

If $\beta=\alpha+\omega$ for some infinite ordinal $\alpha$, then by the induction hypothesis there is a tree with leaves at all infinite levels $\leqslant \alpha$, and hence the desired result follows immediately from Proposition 4.

Suppose $\beta$ is a limit ordinal which is not of the form $\alpha+\omega$. Since $\beta$ is not subtle, there is a closed cofinal set $M \subseteq \beta$ and a function $f$ with $f(\gamma) \subseteq \gamma(\gamma \in M)$ and

$$
\gamma<\gamma^{\prime} \text { implies } f(\gamma) \neq f\left(\gamma^{\prime}\right) \cap \gamma \text {. }
$$

We can clearly assume $\omega \in M$, and further that each ordinal in $M$ is limit: if the cofinality of $\beta$ is not $\omega$, then the set of limit ordinals in $M$ is again closed and cofinal; if $\beta$ has cofinality $\omega$, then there exist limit ordinals $\alpha_{0}<\alpha_{1}<\cdots$ with $\beta=V \alpha_{n}(n \in \omega)$, and we may take $M=\left\{\alpha_{n} \mid n \in \omega\right\}$ and $f\left(\alpha_{n}\right)=n$. Let $\bar{f}(\gamma) \in 2^{\gamma}$ be the characteristic function of $f(\gamma) \subseteq \gamma$. Then

$$
\gamma<\gamma^{\prime} \text { implies } \bar{f}(\gamma) \nsubseteq \bar{f}\left(\gamma^{\prime}\right) \text {. }
$$


Therefore, if we define a subtree $S$ of the uniform binary tree $U=\bigcup_{i \leqslant \beta} 2^{i}$ by $S=\{x \in U \mid \bar{f}(\gamma) \subseteq x$ implies $\bar{f}(\gamma)=x$ for all $\gamma \in M\}$, then each $\bar{f}(\gamma)$ is a leaf of $S$. By Proposition 4 , there is a tree $\bar{S}$ with card $2^{\gamma}$ leaves in $S(\gamma+k)$ for each $\gamma \in M$ and $k<\omega$.

For each $\gamma \in M$, let $\gamma^{\prime}$ be the next larger element in $M$. By the induction hypothesis, there is a binary tree $T_{\gamma}$ with leaves of all infinite depths $\leqslant \gamma^{\prime}$. We extend $\bar{S}$ by identifying one of the leaves in $\bar{S}(\gamma)$ with the root of $T_{\gamma}$ for each $\gamma \in M$. The resulting tree $\tilde{S}$ has a leaf of each depth $i$ with $\omega \leqslant i<\beta$. In fact, let $\gamma \in M$ be the largest ordinal with $\gamma \leqslant i$ (such $\gamma$ exists, since $M$ is closed and $\omega \in M$ ); then $\gamma \leqslant i<\gamma^{\prime}$. Put $i=\gamma+k$; if $k$ is finite, then $S$ has a leaf of depth $\gamma+k$ and hence so does $\bar{S}$ and this leaf is also a leaf in $\tilde{S}$. If $k$ is infinite, $T_{\gamma}$ has a leaf of depth $k$ $\left(<\gamma^{\prime}\right)$ and the corresponding leaf in $\tilde{S}$ has depth $\gamma+k$. The desired tree is shown in the diagram, where $U$ is the uniform binary tree of depth $\beta$.

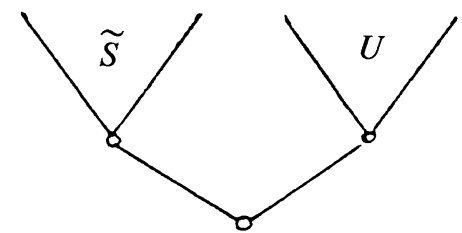

COROLlaRY. There exist arbitrarily large binary trees with leaves of all infinite depths iff no cardinal is subtle. Thus, in particular,

(i) assuming that no cardinal is strongly inaccessible, there exist such trees;

(ii) assuming that such trees exist, no cardinal is measurable.

REMARK. Analogous results hold for $\alpha$-nary trees ( $\alpha$ an infinite cardinal) in place of binary ones. First, the leaves of depths $<\alpha$ are nonimportant: for each $\alpha$-nary tree $S$ there is an $\alpha$-nary tree $S^{\prime}$ with leaves of all depths $<\alpha$, and also of all such depths $\geqslant \alpha$ on which $S$ has a leaf:

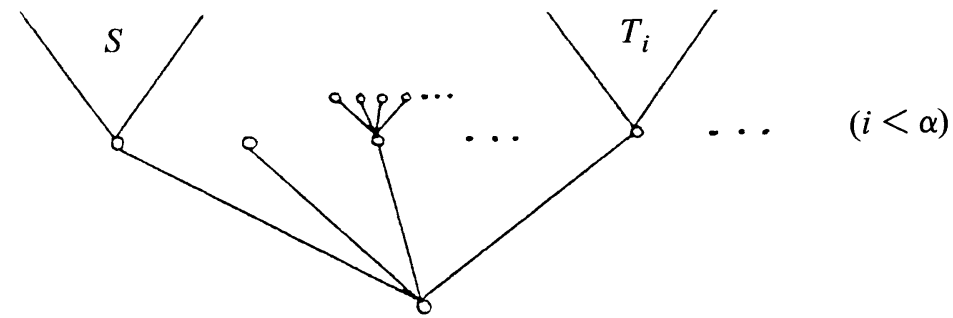

( $T_{i}$ is any tree with a leaf of depth $i$.) Next, the number of leaves of a given depth is unimportant, i.e., Proposition 4 can be readily generalized to the form that there is an $\alpha$-nary tree $\bar{S}$ with card $\alpha^{n}$ leaves of depth $n+k$ for each limit ordinal $n$ such that $S(n)$ contains a leaf, and each finite $k$. The proof of Theorem 1 is now easily generalized (by working, in case of $\alpha$-nary trees, only with leaves of depths $\geqslant \alpha$ ) to the following result.

THEOREM 2. For each infinite cardinal $\alpha$ and each ordinal $\beta>\alpha$ the following are equivalent:

(i) There is an $\alpha$-nary tree with leaves of all nonzero depths $\leqslant \beta$.

(ii) There is a binary tree with leaves of depths $i$ for all $i$ with $\alpha \leqslant i \leqslant \beta$.

(iii) No cardinal $\gamma$ with $\alpha<\gamma \leqslant \beta$ is subtle. 


\section{Unbounded generation.}

4.1. Informal description. We are going to construct a "large" $\Delta_{\omega}$-continuous algebra on one generator, which has just one operation $\sigma$, and that has countable arity. (The adaptation to any other infinitary type is obvious.) Here "large" means of cardinality $\beta$ for each $\beta$ such that there is a binary tree with leaves of all infinite depths $\leqslant \beta$. As proved in $\S 3$, this includes all cardinals $\beta$ for which there are no strongly inaccessible (or no subtle) cardinals below $\beta$. Algebras on more generators are discussed afterwards.

In this section we give an informal explanation of our construction, and the next section contains the formal description and proof.

We start with one generator $x$, and then we generate new points from the old ones (i.e., those already generated) by two means:

(a) applying the operation to $\omega$-tuples of old points;

(b) forming joins of countably-directed sets (e.g., of $n$-chains for ordinals $n$ with cof $n>\omega)$ of old points.

We use a binary tree $S$ with a lot of leaves of all depths. The advantage of a tree is that the structure of all countably-directed subsets is clear: these are just the branches of depth $n$ for cof $n>\omega$. This is important in simplifying the question whether a given operation is $\Delta_{\omega}$-continuous or not.

From the generator $x$ we first create card $2^{\omega}$ points by putting $y=\sigma(x, x, \ldots)$ and letting $\sigma$ be free on all the remaining elements of $\{x, y\}^{\omega}$. These points cover the first $\omega+1$ levels of the tree $S$. Next we would like to generate the level $S(n), n>\omega$, from the previous levels as follows:

(a) $S(n+1)$ is generated from $S(n)$ by the application of $\sigma$; here we use the fact that card $S(n+1)=2 \cdot \operatorname{card} S(n)=\operatorname{card} S(n)$;

(b) $S(n)$ for $n=\mathrm{V}_{k<\omega} n_{k}$ (where $n_{k}<n$ ) is generated from $S\left(n_{0}\right), S\left(n_{1}\right), \ldots$ by the application of $\sigma$ again; here we use the fact that card $S(n)=\operatorname{card} 2^{n}=$ $\Pi_{k<\omega} \operatorname{card} 2^{n_{k}}=$ card $S\left(n_{0}\right) \times S\left(n_{1}\right) \times \cdots$;

(c) $S(n)$ for a limit ordinal with cof $n>\omega$ is generated by joins of $n$-chains: each $s \in S(n)$ is the join of an $n$-chain $s_{i} \in S(i), i<n$, where $s_{i}$ is predecessor of $s$.

This plan has an obstacle: if cof $n>\omega$, then each $\omega$-tuple in $S(n)$ is clearly a join of an $n$-chain of $\omega$-tuples in lower levels. Since $\sigma$ is $\Delta_{\omega}$-continuous, it preserves this $n$-chain join. Thus (a) cannot be performed if $\operatorname{cof} n>\omega$.

We use the following procedure to overcome this difficulty. Instead of a leaf $s \in S$, we work with "additional data" in the form of an $\omega$-sequence

$$
s, s, s, \ldots, s, s_{m}, s_{m+1}, s_{m+2}, \ldots, s_{k}, s_{k}, s_{k}, \ldots,
$$

where $s$ is repeated $m$ times, $s>s_{m}>s_{m+1}>\cdots>s_{k}$ and $s_{k}$ is the root in $S$; the number $m$ is called the width of the $\omega$-sequence. The ordering of these sequences is such that a sequence of width $m+1$ is a $\Delta_{\omega}$-join of sequences of width $m$, and sequences of width 0 are $\Delta_{\omega}$-joins of sequences on lower levels. Thus, sequences of all widths $(<\omega)$ are obtained by successive formation of $\Delta_{\omega}$-joins. But the operation $\sigma\left(z_{0}, z_{1}, \mathrm{z}_{2}, \ldots\right)$ can be defined "freely" if the width of $z_{i}$ grows with $i$ : such $\omega$-tuples $\left(z_{i}\right)_{i<\omega}$ cannot be reached by $\Delta_{\omega}$-joins from $\omega$-tuples on lower levels. 
4.2. Construction of a $\Delta_{\omega}$-continuous algebra of power $\beta$. Let $S$ be a binary tree of depth $\beta$ with card $2^{i}$ leaves of depth $i$ for each infinite $i \leqslant \beta$ (see Proposition 4). We can assume that each chain in $S$ has a join (else, we embed $S$ into the uniform binary tree, and add the missing joins). Consequently, $S$ is $\Delta_{\omega}$-complete.

Let $L \subseteq S$ be the set of all leaves and put

$$
L(i)=L \cap S(i) \quad(i \leqslant \beta) .
$$

Let $C$ be the set of all nonconstant decreasing $\omega$-sequences in $S s: s_{0} \geqslant s_{1} \geqslant s_{2} \geqslant$ ... such that

$$
s_{k}=s_{k+1} \text { implies } s_{k} \text { is either a leaf or the root. }
$$

The first $k$ such that $s_{k}$ is not a leaf will be called the width of $s$ and denoted by $w(s)$. Thus, each $s \in C$ has either the form

$$
s_{0}=s_{1}=\cdots=s_{k-1}>s_{k}>\cdots>s_{n}=s_{n+1}=\cdots
$$

if $k=w(s)>0$, where $s_{0} \in L$ and $s_{n}$ is the root, or the form

$$
s_{0}>s_{1}>\cdots>s_{n}=s_{n+1}=\cdots
$$

if $w(s)=0$, where $s_{0} \in S-L$ and $s_{n}$ is the root.

Denote $C(i)=\left\{s \in C \mid s_{0} \in S(i)\right\}$ for each $i \leqslant \beta$.

Now we define the algebra $A$.

Underlying set: $A=C \cup\{x, y, \perp\}$. Here $x, y, \perp, s$ are pairwise distinct elements for each $s \in C$.

Ordering: For $s, s^{\prime} \in C, s^{\prime} \leqslant s$ iff $s_{k}^{\prime} \leqslant s_{k}$ for $k \leqslant w(s)$, and $s_{k}^{\prime}=s_{k}$ for all $k>w(s)$.

Further $s, x$ and $y$ are pairwise incompatible for each $s \in C$, and $\perp$ is the least element of $A$. Note that $s^{\prime} \leqslant s$ implies $w\left(s^{\prime}\right) \leqslant w(s)$.

Operation: We define $\sigma: A^{\omega} \rightarrow A$ using auxiliary maps $f_{n}(\omega \leqslant n<\beta$, cof $n \leqslant \omega)$ chosen as follows. First, we choose a bijection

$$
f_{\omega}:\{(x, y)\}^{\omega}-\{x\}^{\omega} \rightarrow \bigcup_{n \leqslant \omega} C(n)
$$

which is possible since $S$ is a binary tree (hence, card $S(n) \leqslant \operatorname{card} 2^{n}$ ) and therefore,

$$
\text { card } \bigcup_{n \leqslant \omega} C(n) \leqslant \sum_{n \leqslant \omega}\left(\operatorname{card} 2^{n}\right)^{\omega}=\sum_{n \leqslant \omega} \operatorname{card} 2^{n \omega}=\operatorname{card} 2^{\omega} .
$$

For each isolated ordinal $n>\omega$ we choose a surjective map $f_{n}: L(n-1)^{\omega} \rightarrow C(n)$ which merges almost identical sequences (i.e., $f_{n}\left(s_{0}, s_{1}, s_{2}, \ldots\right)=f_{n}\left(s_{0}^{\prime}, s_{1}^{\prime}, s_{2}^{\prime}, \ldots\right)$ if $s_{k}=s_{k}^{\prime}$ holds for almost all $k$, i.e, for all $k$ larger than some $\left.k_{0} \in \omega\right)$. This is possible because

$$
\operatorname{card} C(n) \leqslant\left(\operatorname{card} 2^{n}\right)^{\omega}=\operatorname{card} 2^{n-1} .
$$

Finally, for each limit ordinal $n$ cofinal with $\omega$ we choose an $\omega$-sequence $n_{k}<n$ with $n=\sum_{k<\omega} n_{k}$, and we choose a surjective map

$$
f_{n}: L\left(n_{0}\right) \times L\left(n_{1}\right) \times L\left(n_{2}\right) \times \cdots \rightarrow C(n)
$$

which merges almost identical sequences. This is possible because

$$
\operatorname{card} C(n) \leqslant \operatorname{card} 2^{n}=\operatorname{card} 2^{\Sigma n_{k}}=\operatorname{card} 2^{n_{0}} \cdot 2^{n_{1}} \cdot 2^{n_{2}} \cdots .
$$


By abuse of language, we evaluate $f_{n}\left(s_{0}, s_{1}, s_{2}, \ldots\right)(n \neq \omega)$ also in the case that all but finitely many $s_{k}$ are in $L(n-1)$ (for $n$ isolated) or in $L\left(n_{k}\right)$ (for cof $n=\omega$ ).

Now we define $\sigma$ as follows: for sequences in $\{x, y\}^{\omega}$ we put

$$
\begin{gathered}
\sigma(x, x, x, \ldots)=y \\
\sigma\left(z_{0}, z_{1}, z_{2}, \ldots\right)=f_{\omega}\left(z_{0}, z_{1}, z_{2}, \ldots\right) \text { if } z_{i} \neq x \text { for some } i .
\end{gathered}
$$

For sequences in $C^{\omega}$ we put

$$
\sigma\left(s^{0}, s^{1}, s^{2}, \ldots\right)=f_{n}\left(s_{0}^{0}, s_{0}^{1}, s_{0}^{2}, \ldots\right)
$$

provided that

(i) the widths of the $s^{k}$ grow beyond all bounds, i.e. $\lim _{k \rightarrow \infty} w\left(s^{k}\right)=\infty$, and

(ii) for almost all $k<\omega$ we have $s_{0}^{k} \in L(n-1)$ for $n$ isolated, $n>\omega$, or $s_{0}^{k} \in L\left(n_{k}\right)$ for $\operatorname{cof} n=\omega$.

All the remaining sequences in $A^{\omega}$ are mapped by $\sigma$ to $\perp$.

THEOREM 3. $A$ is a $\Delta_{\omega}$-continuous algebra generated by $x$.

Proof. (I) $A$ is $\Delta_{\omega}$-complete. It is sufficient to prove that each countably-directed set $M \subseteq C$ has a join. The set $\{w(s) ; s \in M\} \subseteq \omega$ is bounded. (Else, we have $s_{k} \in M$ with $w\left(s_{k}\right) \geqslant k$ for each $k<\omega$, and the countable set $\left\{s_{0}, s_{1}, s_{2}, \ldots\right\}$ has no upper bound in $C$, let alone in $M$.) Let $t \in M$ be an element of the maximal width $k$. Since $M$ is a directed set, we do not lose generality by assuming that $t$ is the least element of $M$. Then $w(s)=k$ for each $s \in M$ and, moreover, $t_{i}=s_{i}$ for each $i \in \omega-\{k\}, s \in C$. Put

$$
s_{k}^{*}=\bigvee_{s \in M} s_{k} \text { in } S
$$

(this join exists because $S$ is a tree with joins of all chains and hence, with joins of all directed subsets). Then obviously

$$
\bigvee M=\left(t_{0}, t_{1}, \ldots, t_{k-1}, s_{k}^{*}, t_{k+1}, \ldots\right) \text {. }
$$

(II) For each $\omega$-directed set $M \subseteq C$ we have

$$
w(\bigvee M) \leqslant \bigvee_{s \in M} w(s)+1
$$

This follows from the above description of the joins.

(III) The operation $\sigma$ is order-preserving. In fact, for $p<q$ in $A^{\omega}$ with $\sigma(p) \neq \perp$ we prove that $\sigma(q)=\sigma(p)$. This is clear for $p \in\{x, y\}^{\omega}$ because $p<q$ does not hold for any $q$.

Let $p=\left(s^{k}\right)$ with $\lim _{k \rightarrow \infty} w\left(s^{k}\right)=\infty$. Then $p<q$ implies $q=\left(t^{k}\right)$ with $\lim w\left(t^{k}\right)=\infty$. Moreover, $s_{0}^{k} \leqslant t_{0}^{k}$ and hence, if $s_{0}^{k}$ is a leaf, then $s_{0}^{k}=t_{0}^{k}$. Since $\sigma(p) \neq \perp$, this holds for all but finitely many $k<\omega$ and hence,

$$
\boldsymbol{\sigma}(p)=f_{n}\left(s_{0}^{0}, s_{0}^{1}, s_{0}^{2}, \ldots\right)=f_{n}\left(t_{0}^{0}, t_{0}^{1}, t_{0}^{2}, \ldots\right)=\sigma(q) .
$$

(IV) $\sigma$ is $\Delta_{\omega}$-continuous. The only nontrivial case is when $M \subseteq C^{\omega}$ is a countablydirected set with a join

$$
\bigvee M=\left(t^{0}, t^{1}, t^{2}, \ldots\right) \quad \text { with } \sigma\left(t^{0}, t^{1}, t^{2}, \ldots\right) \neq \perp
$$


Then we prove that $\sigma(\bigvee M)=\bigvee \sigma(M)$ by finding $s \in M$ with $\sigma\left(s^{0}, s^{1}, s^{2}, \ldots\right)=$ $\sigma\left(t^{0}, t^{1}, t^{2}, \ldots\right)$.

Since $\lim _{k \rightarrow \infty} w\left(t^{k}\right)=\infty$, for each $i<\omega$ there is $i^{*}<\omega$ with $w\left(t^{k}\right)>i+1$ for each $k \geqslant i^{*}$. Since

$$
t^{k}=\bigvee_{s \in M} s^{k},
$$

it follows from (II) above that $w\left(s^{k}\right)>i$ for some $s \in M$; this $s$ depends on $i<\omega$ and $k$ with $i^{*} \leqslant k<\omega$, and we denote it by $s(i, k)$. Let $s \in M$ be an upper bound of the countable set of all $s(i, k)$ for $i \in \omega, k \in \omega-i^{*}$. Then $w\left(s^{k}\right)>i$ for each $k \geqslant i^{*}$ and hence, $\lim _{k \rightarrow \infty} w\left(s^{k}\right)=\infty$. Moreover, each $s_{0}^{k}$ with $k \geqslant 0 *$ is a leaf (because $w\left(s_{0}^{k}\right)>0$ is the number of leaves in $s$ ) and thus,

$$
s_{0}^{k} \leqslant t_{0}^{k} \text { implies } s_{0}^{k}=t_{0}^{k} \text { for each } k \geqslant 0 * .
$$

Consequently,

$$
\sigma\left(t^{0}, t^{1}, t^{2}, \ldots\right)=f_{n}\left(t_{0}^{0}, t_{0}^{1}, t_{0}^{2}, \ldots\right)=f_{n}\left(s_{0}^{0}, s_{0}^{1}, s_{0}^{2}, \ldots\right)=\sigma\left(s^{0}, s^{1}, s^{2}, \ldots\right) .
$$

(V) $A$ is generated by $x$. We prove that each ( $\Delta_{\omega}$-continuous) subalgebra $B \subseteq A$ containing $x$ is equal to $A$. First, $y=\sigma(x, x, x, \ldots) \in B$ because $B$ is closed under $\sigma$, and $\perp=\mathrm{V}_{\phi} \in B$ because $B$ is closed under $\Delta_{\omega}$-joins. We prove $C(n) \subseteq B$ by induction.

(a) First $\omega+1$ steps:

$$
\bigcup_{n \leqslant \omega} C(n) \subseteq \sigma\left(\{x, y\}^{\omega}\right) \subseteq \sigma\left(B^{\omega}\right) \subseteq B .
$$

(b) Isolated step: If $C(n-1) \subseteq B$ for $n>\omega$, then for each $s \in C(n)$ we have $s=f_{n}\left(s_{0}^{0}, s_{0}^{1}, s_{0}^{2}, \ldots\right)$ for some $s_{0}^{k} \in L(n-1)$. Define $s^{k}=\left(s_{0}^{k}, s_{0}^{k}, \ldots, s_{0}^{k}, r, r, r, \ldots\right)$ $\in C(n-1)$, where $s_{0}^{k}$ is repeated $k$ times, and $r$ is the root in $S$. Then clearly

$$
s=f_{n}\left(s_{0}^{0}, s_{0}^{1}, s_{0}^{2}, \ldots\right)=\sigma\left(s^{0}, s^{1}, s^{2}, \ldots\right) \in \sigma\left(C(n-1)^{\omega}\right) \subseteq \sigma\left(B^{\omega}\right) \subseteq B .
$$

(c) Limit step: If $C(m) \subseteq B$ for all $m<n$, where $n$ is a limit ordinal, we prove $C(n) \subseteq B$. The argument in case cof $n=\omega$ is the same as in the isolated step.

Let $\operatorname{cof} n>\omega$. Put for each $k<\omega$

$$
C(n \mid k)=\left\{s \in \bigcup_{m \leqslant n} C(m) \mid s_{i} \in \bigcup_{m<n} S(m) \text { for all } i \geqslant k\right\} .
$$

Then $C(n) \subseteq \cup_{k<\omega} C(n \mid k)$ and we prove $C(n \mid k) \subseteq B$ by induction in $k . C(n \mid 0) \subseteq B$ by the induction hypothesis (for $n$ ).

Let $C(n \mid k) \subseteq B$ and let $s \in C(n \mid k+1)-C(n \mid k)$. Then

$$
s=\left(s_{0}, s_{0}, \ldots, s_{0}, s_{1}, s_{2}, \ldots\right),
$$

where $s_{0} \in S(n)$ is repeated $k+1$ times, and $s_{0}>s_{1}$. Then

$$
s_{0}=\bigvee\left\{t \in S \mid s_{1}<t<s_{0}\right\}
$$

in $S$ and since cof $n>\omega$, this is a $\Delta_{\omega}$-join. For each $t$ put $s^{t}=\left(s_{0}, s_{0}, \ldots, s_{0}, t\right.$, $\left.s_{1}, s_{2}, \ldots\right) \in C(n \mid k)$, where $s_{0}$ is repeated $k$ times. Then $s=\mathrm{V}_{s_{1}<t<s_{0}} s^{t}$ is a $\Delta_{\omega}$-join in $A$. Since $s^{t} \in B$ by the induction hypothesis (for $k$ ), we get $s \in B$. 
REMARK. For more than one generator, we proceed as in the preceding construction with an $\alpha$-ary tree instead of a binary tree. Assume that $S$ is a tree with $\alpha^{i}$ leaves of depth $i$ for each $0<i \leqslant \beta$. (Such a tree exists iff no cardinal between $\alpha$ and $\beta$ is subtle, see Theorem 2 in §3.) Then we use the root and the nodes in $S(1)$ as the set of generators, thus disposing of $x$ and $y$ in the construction above. Otherwise, the construction is unchanged, except that the maps $f_{n}$ are defined for all $n \geqslant 1$. This proves the following result.

THEOREM 4. If there is no subtle cardinal $\gamma$ with $\alpha<\gamma \leqslant \beta$, then there is $a$ $\Delta_{\omega}$-continuous algebra of power $\beta$ with $\alpha$ generators.

COROLlARY. If there exists a cardinal larger than all strongly inaccessible cardinals, then generation of infinitary $\Delta_{\omega}$-continuous algebras is unbounded.

5. Unbounded strong generation. Here we strengthen the results of the preceding section. We again start with a cardinal $\beta$ for which there is a binary tree $S$ with $2^{i}$ leaves of depth $i$ for all infinite $i \leqslant \beta$. We now construct a $\Delta_{\omega}$-continuous algebra of power $\beta$ which is strongly generated by one element. The present construction is substantially more complicated than the previous one.

Notice that for the algebra from $\S 4$, the weak subalgebra strongly generated by the generator $x$ is rather small; it is the algebra $A_{0}=\{x, y, \perp\} \cup \cup_{n \leqslant \omega} C(n)$ with the discrete order except for $\perp$ remaining the least element; the cardinality of $A_{0}$ is card $2^{\omega}$. To obtain strong generation, we modify the construction of $\S 4$ to strengthen the generation principles as follows:

(1) The operation creates not only new points but also their order (via $\sigma\left(a_{i}\right) \leqslant \sigma\left(b_{i}\right)$ if $a_{i} \leqslant b_{i}$ for all $i$ ).

(2) New points obtained as joins of $\Delta_{\omega}$-directed sets are created with their order (viz $\vee a_{i} \leqslant \bigvee b_{i}$ if $a_{i} \leqslant b_{i}$ for all $i$ ).

Construction. We keep the notation $S(n), L$ etc. of $\S 4$.

Let $\tilde{S}=(S \times\{0,1\})-(L \times\{0\})$ with component-wise ordering where $0 \leqslant 1$. For $s=(t, i)$ put $p_{1} s=t, p_{2} s=i$. $\tilde{S}$ is $\Delta_{\omega}$-complete: for each countably directed $M \subseteq \tilde{S}$, let $t=\bigvee_{s \in M} p_{1} s$ in the tree $S$. Then

$$
\bigvee M= \begin{cases}(t, 0) & \text { if } t \in L \text { and } p_{2} s=0 \text { for all } s \in M, \\ (t, 1) & \text { else. }\end{cases}
$$

For example, for each $t \in L$,

$$
(t, 1)=\bigvee_{t^{\prime}<t}\left(t^{\prime}, 0\right)=\bigvee_{t^{\prime}<t}\left(t^{\prime}, 1\right)
$$

Notice that $\tilde{S}$ is a tree no more; nevertheless, $s \in \tilde{S}$ will be called a leaf or a root if $p_{1} s$ is a leaf or a root, respectively. $\tilde{L}$ will denote the set of all leaves in $\tilde{S}$, and $\tilde{L}(n)=\tilde{S}(n) \cap \tilde{L}$, where $\tilde{S}(n)=\left\{s \in \tilde{S} \mid p_{1} s \in S(n)\right\}$.

Denote by $\tilde{C}$ the set of all nonconstant decreasing $\omega$-sequences in $\tilde{S}$,

$$
s: s_{0} \geqslant s_{1} \geqslant s_{2} \geqslant \cdots,
$$

such that $s_{k}=s_{k+1}$ implies $s_{k}$ is either a leaf or a root. Again, the first $k$ with $s_{k}$ not a leaf will be called the width of $s$ and will be denoted by $w(s)$. For each $n \leqslant \beta$ put $\tilde{C}(n)=\left\{s \in \tilde{C} \mid s_{0} \in \tilde{S}(n)\right\}$. Now we are ready to define our algebra $\tilde{A}$. 
Underlying set: $\tilde{A}=\tilde{C} \cup\{x, y, \perp\}$, where $x, y, \perp, s$ are distinct elements for each $s \in \tilde{C}$.

Ordering: For $s, s^{\prime} \in \tilde{C}, s^{\prime} \leqslant s$ iff $s_{k}^{\prime} \leqslant s_{k}$ for all $k<\omega$ and $k \geqslant w(s)$,

$$
p_{2} s_{k}^{\prime}=p_{2} s_{k} \text { implies } s_{i}=s_{i}^{\prime} \text { for all } i>k .
$$

Further, $s, x, y$ are pairwise incomparable for each $s \in \tilde{C}$, and $\perp$ is the least element of $\tilde{A}$.

Operation: We make use of auxiliary surjective maps $f_{n}$ :

For $n=\omega$,

$$
f_{\omega}:\{x, y\}^{\omega}-\{x\}^{\omega} \rightarrow\left\{(a, b) \mid a, b \in \bigcup_{n \leqslant \omega} \tilde{C}(n), a \leqslant b\right\} .
$$

For isolated $n>\omega$,

$$
f_{n}: \tilde{L}(n-1)^{\omega} \rightarrow\left\{(a, b) \mid a, b \in \bigcup_{m \leqslant n} \tilde{C}(m), a \leqslant b\right\} .
$$

For limit $n>\omega$ with cof $n=\omega$, where $n_{1}, n_{2}, \ldots$ is a fixed increasing sequence with $\mathrm{V}_{i<\omega} n_{i}=n$,

$$
f_{n}: \tilde{L}\left(n_{1}\right) \times \tilde{L}\left(n_{2}\right) \times \cdots \rightarrow\left\{(a, b) \mid a, b \in \bigcup_{m \leqslant n} \tilde{C}(m), a \leqslant b\right\} .
$$

We suppose, as in 4.2 , that the maps $f_{n}(n \neq \omega)$ merge almost identical sequences; each of them will be applied also to sequences $s_{0}, s_{1}, s_{2}, \ldots$ such that all but finitely many $s_{k}$ are in $\tilde{L}(n-1)$ (for $n$ isolated) or in $\tilde{L}\left(n_{k}\right)$ (for $n$ limit with cof $n=\omega$ ).

We put $\sigma(x, x, x, \ldots)=y$, and for all other sequences $\left(z_{0}, z_{1}, z_{2}, \ldots\right)$ in $\{x, y, \perp\}^{\omega}$ we put

$$
\sigma\left(z_{0}, z_{1}, z_{2}, \ldots\right)= \begin{cases}\perp & \text { if } z_{2 n}=\perp \text { for some } n<\omega \\ a & \text { if } z_{2 n+1}=\perp \text { and } z_{2 n} \neq \perp \text { for all } n<\omega \\ b & \text { if } z_{2 n+1} \neq \perp \text { for some } n<\omega \text { and } z_{2 n} \neq \perp \text { for all } n<\omega\end{cases}
$$

where $(a, b)=f_{\omega}\left(z_{0}, z_{2}, z_{4}, \ldots\right)$.

For sequences in $(\tilde{C} \cup\{\perp\})^{\omega}$ put

$$
\sigma\left(s^{0}, s^{1}, s^{2}, \ldots\right)= \begin{cases}a & \text { if } s^{2 n+1}=\perp \text { for all } n<\omega, \\ b & \text { if } s^{2 n+1} \neq \perp \text { for some } n<\omega,\end{cases}
$$

where $(a, b)=f_{n}\left(s_{0}^{0}, s_{0}^{2}, s_{0}^{4}, \ldots\right)$, provided that

(i) $\lim _{k \rightarrow \infty} w\left(s^{2 k}\right)=\infty$, and

(ii) for almost all $k<\omega$ we have

$$
\begin{gathered}
s_{0}^{2 k} \in \tilde{L}(n-1) \text { for } n \text { isolated, } n>\omega, \\
s_{0}^{2 k} \in \tilde{L}\left(n_{k}\right) \text { for } n \text { limit, } \operatorname{cof} n=\omega .
\end{gathered}
$$

All the remaining sequences in $\tilde{A}^{\omega}$ are mapped by $\sigma$ to $\perp$.

THEOREM 5. $\tilde{A}$ is a $\Delta_{\omega}$-continuous algebra strongly generated by $x$. 
Proof. The proof of the $\Delta_{\omega}$-completeness of $\tilde{A}$ is the same as that for $A$ in the proof of Theorem 3. Also, the proof of $\Delta_{\omega}$-continuity of the operation is analogous. Therefore, we restrict ourselves to the proof that $x$ strongly generates $\tilde{A}$.

Let $B$ be a weak subalgebra of $\tilde{A}$ with the ordering denoted by $\sqsupseteq$. We prove that if $x \in B$, then $B=\tilde{A}$ and $\sqsupseteq$ coincides with $\leqslant$.

Given $a, b \in \tilde{A}$ with $a \leqslant b$, it is our task to prove that $a, b \in B$ and $a \sqsupseteq b$. This is obvious if either $a$ or $b \in\{x, y, \perp\}$. Hence, we can suppose that $a, b \in \bigcup_{m \leqslant n} \tilde{C}(m)$ for some $n$ and we proceed by induction on $n$.

(i) Let $n \leqslant \omega$. There is $\left(z_{0}, z_{1}, z_{2}, \ldots\right) \in\{x, y\}^{\omega}-\{x\}^{\omega}$ with $f_{\omega}\left(z_{0}, z_{1}, z_{2}, \ldots\right)=$ $(a, b)$ and then

$$
\begin{aligned}
& a=\sigma\left(z_{0}, \perp, z_{1}, \perp, z_{2}, \perp, \ldots\right), \\
& b=\sigma\left(z_{0}, x, z_{1}, x, z_{2}, x, \ldots\right) .
\end{aligned}
$$

Since $x \in B$ implies $y=\sigma(x, x, x, \ldots) \in B$ and $\perp=\bigvee \varnothing \in B$, we conclude

$$
\sigma\left(\{x, y, \perp\}^{\omega}\right) \subseteq B
$$

hence $a, b \in B$. Since $\sigma$ is order-preserving and $\perp=\vee \varnothing \sqsupseteq x$, we have $a \sqsupseteq b$.

(ii) Assuming the proposition holds for some $n \geqslant \omega$, consider $a, b \in \bigcup_{m \leqslant n+1} \tilde{C}(m)$. Then there is $\left(s_{0}^{0}, s_{0}^{1}, s_{0}^{2}, \ldots\right) \in L(n)^{\omega}$ with $f_{n+1}\left(s_{0}^{0}, s_{0}^{1}, s_{0}^{2}, \ldots\right)=(a, b)$. Define $s^{i} \in$ $\tilde{C}(n)$ by

$$
s^{i}=\left(s_{0}^{i}, s_{0}^{i}, \ldots, s_{0}^{i}, r, r, \ldots\right),
$$

where $s_{0}^{i}$ is repeated $i$ times and $r<s_{0}^{i}$ is a root. Then

$$
\begin{aligned}
& a=\sigma\left(s^{0}, \perp, s^{1}, \perp, s^{2}, \perp, \ldots\right), \\
& b=\sigma\left(s^{0}, s^{0}, s^{1}, s^{1}, s^{2}, s^{2}, \ldots\right) .
\end{aligned}
$$

Since $s^{i} \in \tilde{C}(n) \subseteq B$ and $\perp \in B$, we have $a, b \in \sigma\left(B^{\omega}\right) \subseteq B$. Also $a \sqsupseteq b$ as above.

(iii) Assuming that $n>\omega$ is a limit ordinal and that the proposition holds for all $m<n$, consider $a, b \in \bigcup_{m \leqslant n} \tilde{C}(m)$. We may assume $b \in \tilde{C}(n)$. If cof $n=\omega$, we proceed as in (ii) above. Let cof $n>\omega$. We proceed by induction on $w(b)$.

(a) Let $w(b)=0$. Then also $w(a)=0$ and we can write

$$
a=\left(\left(t^{\prime}, u\right), a_{1}, a_{2}, \ldots\right), \quad b=\left((t, v), b_{1}, b_{2}, \ldots\right),
$$

where $u \leqslant v$ in $\{0,1\}, t^{\prime} \leqslant t \in \tilde{S}(n)$ and $a_{i} \leqslant b_{i}$ in $\bigcup_{m \leqslant n} \tilde{S}(m)$ for $i>1$.

(a1) Let $a \in \cup_{m<n} \tilde{C}(m)$. Then we have a $\Delta_{\omega}$-join (viz, a join of an $n$-chain) in $\tilde{A}$,

$$
b=\bigvee_{t^{\prime}<s<t}\left((s, v), b_{1}, b_{2}, \ldots\right) \text {. }
$$

Also, $a \leqslant\left((s, v), b_{1}, b_{2}, \ldots\right)$ for all $s$ with $t^{\prime}<s<t$. By the induction hypothesis, the elements $\left((s, v), b_{1}, b_{2}, \ldots\right) \in \bigcup_{m<n} \tilde{C}(m)$ are in $B$ and form a chain with respect to the ordering $\sqsubseteq$, and $a \sqsubseteq\left((s, v), b_{1}, b_{2}, \ldots\right)$ for $t^{\prime}<s<t$. As the inclusion map $B \rightarrow \tilde{A}$ is supposed to be $\Delta_{\omega}$-continuous, we can conclude $b \in B$ and $a \sqsubseteq b$.

(a2) Let $a \in \tilde{C}(n)$. Then $t^{\prime}=t$ and we have (using the same argument as above)

$$
a=\bigvee_{s<t}\left((s, u), a_{1}, a_{2}, \ldots\right), \quad b=\bigvee_{s<t}\left((s, v), b_{1}, b_{2}, \ldots\right)
$$


with respect to the ordering $\sqsubseteq$. Therefore $a, b \in B$. Clearly $\left((s, u), a_{1}, a_{2}, \ldots\right) \leqslant b$ for $s<t$. Hence, by (a1), $\left((s, u), a_{1}, a_{2}, \ldots\right) \sqsubseteq b$ (for $\left.s<t\right)$ and so $a \sqsubseteq b$.

(b) Let $w(b)=k>0$. We proceed by induction on $w(b)-w(a)$.

(b1) Let $w(b)-w(a)=0$. We can write

$$
\begin{aligned}
& a=\left((t, 1),(t, 1), \ldots,(t, 1),\left(t_{k}^{\prime}, n_{k}^{\prime}\right),\left(t_{k+1}^{\prime}, n_{k+1}^{\prime}\right), \ldots\right), \\
& b=\left((t, 1),(t, 1), \ldots,(t, 1),\left(t_{k}, n_{k}\right),\left(t_{k+1}, n_{k+1}\right), \ldots\right)
\end{aligned}
$$

for some $t \in L(n)$ and $\left(t_{i}^{\prime}, n_{i}^{\prime}\right) \leqslant\left(t_{i}, n_{i}\right)$ in $\bigcup_{m<n} \tilde{S}(m)$ such that $n_{i}=n_{i}^{\prime}$ implies $\left(t_{j}^{\prime}, n_{j}^{\prime}\right)=\left(t_{j}, n_{j}\right)$ for all $j>i$. Then we have

$$
a=\bigvee_{s<t} a^{s}, \quad b=\bigvee_{s<t} b^{s} \quad(\text { in } A),
$$

where

$$
\begin{aligned}
& a^{s}=\left((t, 1),(t, 1), \ldots,(t, 1),(s, 0),\left(t_{k}^{\prime}, n_{k}^{\prime}\right),\left(t_{k+1}^{\prime}, n_{k+1}^{\prime}\right), \ldots\right), \\
& b^{s}=\left((t, 1),(t, 1), \ldots,(t, 1),(s, 1),\left(t_{k}, n_{k}\right),\left(t_{k+1}, n_{k+1}\right), \ldots\right) .
\end{aligned}
$$

Clearly $a^{s} \leqslant b^{s}$ for all $s<t$. As $w\left(a^{s}\right)=w\left(b^{s}\right)=k-1$, using the induction assumption we get $a, b \in B, a \sqsubseteq b$ by the same argument as in (a).

(b2) Let $w(b)-w(a)>0$. Put $w(a)=r \geqslant 0$. Then we can write

$$
\begin{aligned}
a & =\left((t, 1),(t, 1), \ldots,(t, 1),\left(t^{\prime}, u\right), a_{r+1}, a_{r+2}, \ldots\right), \\
b & =\left((t, 1),(t, 1), \ldots,(t, 1),(t, 1), \ldots,(t, 1), b_{k}, b_{k+1}, \ldots\right) .
\end{aligned}
$$

Put $a^{s}=\left((t, 1),(t, 1), \ldots,(t, 1),(s, 1), a_{r+1}, a_{r+2}, \ldots\right)$ for $t^{\prime}<s \leqslant t$ and $a^{\prime}=a^{t}$. Then $a^{\prime}=\bigvee_{t^{\prime}<s<t} a^{s}$ in $\tilde{A}$, and $a \leqslant a^{\prime} \leqslant b$.

Since $w\left(a^{s}\right)=w(a)<w(b)$ for all $s$ with $t^{\prime}<s<t$, we can use the induction hypothesis in $k$ to conclude that elements $a^{s}\left(t^{\prime}<s<t\right)$ form a chain with uncountable cofinality in $B$ with respect to the ordering $\sqsubseteq$ and $a \sqsubseteq a^{s}$ for all $s$ with $t^{\prime}<s<t$. It follows $a^{\prime} \in B$ and $a \sqsubseteq a^{\prime}$. Finally, since $w\left(a^{\prime}\right)=r+1$, we have $w(b)-w\left(a^{\prime}\right)<w(b)-w(a)$. Consequently, $a^{\prime} \leqslant b$ implies $b \in B, a^{\prime} \sqsubseteq b$ by the induction hypothesis in $w(b)-w(a)$. Therefore $a, b \in B, a \sqsubseteq b$. The proof is finished.

REMARK. The preceding result can be generalized to $\alpha$ generators analogously as in the case of Theorems 3 and 4 :

THEOREM 6. If $\alpha$ is a cardinal larger than all subtle cardinals, then there exist arbitrarily large $\Delta_{\omega}$-continuous algebras strongly generated by $\alpha$ generators.

COROLlaRY. Assume that there exists a cardinal larger than all strongly inaccessible cardinals. Then for each infinitary type $\Sigma$

(i) strong generation of $\Delta_{\omega}$-continuous algebras is unbounded;

(ii) the category of $\Delta_{\omega}$-continuous algebras is not extremally co-well-powered.

\section{REFERENCES}

1. J. Adámek, Colimits of algebras revisited, Bull. Austral. Math. Soc. 17 (1977), 433-450.

2. Construction of free continuous algebras, Algebra Universalis 14 (1982), 140-166.

3. J. Adámek and E. Nelson, Separately continuous algebras, Theoret. Comput. Sci. 27 (1983), $225-231$.

4. J. Adámek, E. Nelson and J. Reiterman, Tree constructions of free continuous algebras, J. Comput. System Sci. 24 (1982), 114-146. 
5. ADJ (J. W. Thatcher, E. G. Wagner and J. B. Wright), A uniform approach to inductive posets and inductive closure, Theoret. Comput. Sci. 7 (1978), 57-77.

6. B. Banaschewski and E. Nelson, Completions of partially ordered sets, SIAM J. Comput. 11 (1982), 521-528.

7. J. E. Baumgartner, Ineffability properties of cardinals. I, Infinite and Finite Sets, Colloq. Math. Soc. János Bolyai 10 (1975), 109-130.

8. N. Bourbaki, Theorie des ensembles, Chapter 4, Structures, Actualités Sci. Indust., no. 1253, Hermann, Paris, 1957.

9. H. Herrlich and G. E. Strecker, Category theory, Allyn \& Bacon, Boston, Mass., 1973.

10. A. Kanamori and M. Magidor, The evolution of large cardinal axioms in set theory, Lecture Notes in Math., vol. 669, Springer-Verlag, Berlin and New York, 1978, pp. 99-276.

11. E. Nelson, Free Z-continuous algebras, Lecture Notes in Math., vol. 871, Springer-Verlag, Berlin and New York, 1981, pp. 315-334.

12. A. Pasztor, Epis in some categories of Z-continuous partial algebras, IFI-Report 5A/81, Stuttgart, Acta. Cybernet. (to appear).

Faculty of Electrical Engineering, Technical University, Prague, Czechoslovakia

Faculty of Mathematics and Physics, Charles University, Prague, Czechoslovakia

Department of Mathematics, McMaster University, Hamilton, Ontario, Canada L8S 4K1

Faculty of Nuclear Physics, Technical University, Prague, Czechoslovakia 